FERMILAB-Pub-82/80 2000.000

A DETERMINATION OF INTRINSIC TRANSVERSE MOMENTUM OF QUARKS

P. K. Malhotra and R. Orava

November 1982 


\title{
A DETERMINATION OF
}

INTRINSIC TRANSVERSE MOMENTUM OF QUARKS

\author{
P.K. Malhotra* and $\mathrm{R}$. Orava** \\ Fermi National Accelerator Laboratory \\ P.O. Box 500 \\ Batavia, IL 60510
}

\section{Abstract}

It is suggested that the observed universality of the transverse momentum squared distribution for the meson resonances $n, \rho, \omega, f$ and $K^{*}$ in soft hadron-hadron interactions arises because they are dominantly produced as a result of quark-antiquark fusion (recombination). The universal value of the slope parameter is then interpreted as due to the intrinsic transverse momentum $\left(k_{T}\right)$ of the quarks which yields the values $\left\langle\mathrm{k}_{\mathrm{T}}\right\rangle_{\mathrm{q}}=0.36 \pm 0.01 \mathrm{GeV} / \mathrm{c}$ and $\left\langle\mathrm{k}_{\mathrm{T}}^{2}\right\rangle_{\mathrm{q}}=0.16 \pm 0.01(\mathrm{GeV} / \mathrm{c})^{2}$.

* On leave from the Tata Institute of Fundamental Research, Bombay 400-005, India.

** R.R. Wilson Fellow at Fermilab, on leave from the University of Helsinki, Helsinki, Finland. 


\section{Introduction}

The fact that partons are confined in a hadron of limited dimensions would lead one to expect that they must have a certain amount of intrinsic motion $(1,2)$. So far there is no prediction of the distribution of the intrinsic transverse momentum $\left(k_{T}\right)$ on the basis of QCD. There are however a number of papers(3) in which the dependence of $k_{\mathrm{T}}$ on the Feynman $x$ has been explored and growth of $\left\langle\mathrm{k}_{\mathrm{T}}>\right.$ with $\mathrm{Q}^{2}$ has been argued on physical grounds $(4)$.

At the phenomenological level, it has been increasingly apparent in recent years that in order to understand the transverse momentum distributions of hadrons produced in proton-proton interactions and in lepton-nucleon interactions or the transverse momentum distribution of high mass muon pairs produced in hadron-hadron interactions, it is necessary to invoke an intrinsic transverse momentum distribution for the partons. Feynman et al.(1) proposed a gaussian distribution for the parton $k_{\mathrm{T}}$ with $\left\langle\mathrm{k}_{\mathrm{T}}>\sim 850 \mathrm{MeV} / \mathrm{c}\right.$. These and other authors(5) find that in order to understand the inclusive pp data on the basis of the scale violation effects inherent in the asymptotically free $Q C D$, it is necessary to invoke such a large intrinsic $<k_{\mathrm{T}}>$. Similarly, parton $k_{T}$ smearing has also been introduced $(6)$ to regularize QCD prediction and to improve agreement with the observed Drell-Yan muon pair transverse momentum distribution. We have recentIy carried out a similar analysis(7) of all the available data on high mass dimuon production in $\mathrm{pN}, \overline{\mathrm{p}} \mathrm{N}$ and $\pi^{-} \mathrm{N}$ interactions on the basis of first order QCD formalism and find agreement only when 
large $k_{\mathrm{T}}\left(\left\langle\mathrm{k}_{\mathrm{T}}^{2}\right\rangle=0.9\right.$ to $\left.1.5(\mathrm{GeV} / \mathrm{C})^{2}\right)$ smearing is included. Caswell and Horgan and Brodsky(8) have however pointed out that there are serious theoretical difficulties in incorporating such a large constituent $k_{T}$ fluctuations into the parton model.

In this paper we have estimated the primordial < $\left.\mathrm{k}_{\mathrm{T}}\right\rangle$ from the $\mathrm{p}_{\mathrm{T}}$ distribution of the meson resonances produced in hadron-hadron interactions.

2. Universality of the transverse momentum distributions of the prompt hadrons

A few years ago we made an observation(9) that in hadron-hadron collisions the $\mathrm{p}_{\mathrm{T}}$ distributions of the directly produced mesons such as $\eta, \rho^{\circ}, \omega, f$ and $k^{*}(890)$ exhibit a striking universality of the form

$$
\frac{d \sigma}{d p_{T}^{2}}=A \cdot \exp \left(-B p_{T}^{2}\right)
$$

for $p_{\mathrm{T}}^{2} \lesssim 2(\mathrm{GeV} / \mathrm{C})^{2}$ with $\mathrm{B}=3.4 \pm 0.2(\mathrm{GeV} / \mathrm{c})^{-2}$.

Support for the universality of the slope parameter $B$ was reviewed by $\mathrm{kittel}$ in $1977(10)$. In recent years a considerable amount of additional data has accumulated in the literature and these support the universality hypothesis. One of us has carried out(11) an upto date compilation of the B parameter for $\eta, \rho, \omega$, 
E, $K^{*}(890)$ and $\phi$ produced in the $\pi^{ \pm} p, p p, \bar{p} p$ and $K^{ \pm} p$ interactions from $16 \mathrm{GeV} / \mathrm{c}$ to the ISR energies. The essential results of this compilation are that the values of $B$ are independent of the mass of the meson and the weighted mean values of $B$ are found to be $3.2 \pm 0.2$ for $\eta, \rho^{\circ}, \rho^{ \pm}, \omega$ and $f, 3.0 \pm 0.2$ for $k^{\star 0}, \bar{K}^{\star 0}$ and $K^{\star \pm}$ and $3.0 \pm 0.2$ for the $\phi$ meson. The errors reflect essentially the systematic uncertainties. Since these values reflect no dependence on the mass or the quark content of the mesons, we give below the grand weighted mean value:

$$
\mathrm{B}=3.1 \pm 0.2
$$

This value implies that $\left\langle\mathrm{p}_{\mathrm{T}}^{2}\right\rangle=0.323(\mathrm{GeV} / \mathrm{C})^{2}$ and $\left\langle\mathrm{p}_{\mathrm{T}}\right\rangle=0.504 \mathrm{GeV} / \mathrm{c}$ for the light quark meson resonances.

The following remarks are relevant here:

(i) It should be noted that $B$ is independent of the mass of the resonance over a fairly broad range, i.e. $0.549 \mathrm{GeV}$ for $n$ to $1.27 \mathrm{GeV}$ for the $\mathrm{f}$.

(ii) The ground state mesons, namely the pions and the kaons do not show this universality. It was found (9) for example that the pion $\mathrm{p}_{\mathrm{T}}^{2}$ distribution falls more steeply from $\mathrm{p}_{\mathrm{T}}^{2}=0$ to $1(\mathrm{GeV} / \mathrm{c})^{2}$ but joins the $\rho^{\circ}$ and $f$ trend at larger $p_{T}^{2}$. similar observation was also made for the kaons (12). Also it was shown that pions and kaons arise dominantly through the decay of the resonances (9,13-19). These observations lead to the conclusion that pions. and kaons have two components, a direct and an indirect one with only the direct component obeying the universality. 
(iii) It is interesting to note that $\phi$ meson too has the same value for $B$ as observed for the non-strange and strange mesons. However, it is well known that the $J / \psi$ and the upsilon have much flatter $p_{T}$-distributions. These features would be commented upon in the next section.

3. Primordial transverse momentum of quarks

The universality of the $\mathrm{p}_{\mathrm{T}}^{2}$ distribution of the directly produced mesons can be qualitatively understood in the framework of naive quark model (20). In this picture one of the quarks from the incident hadron interacts with a quark in the target hadron leading to the production of a number of quark-antiquark pairs. The incident quarks both in the projectile and the target hadrons remain "spectators", which eventually combine with newly created quarks and antiquarks to form hadrons in the fragmentation region. The remaining quarks generally have smaller momenta in the c.m.s and combine to form hadrons in the central region. In this picture the transverse momentum distribution of the created hadrons will be determined essentially by the intrinsic $k_{T}$ of the quarks and antiquarks. It follows therefore that the $p_{T}$ distribution of the mesons which are dominantly produced through the $q \bar{q}$ fusion in this manner will have similar shapes independent of the mass of the meson.

In principle this picture could be sightly modified by the soft gluon effects (we are restricting ourselves to the soft hadronic interactions implying low $Q^{2}$ for the sub processes) The 
near constancy of the slope parameter $\mathrm{B}$ for $\mathrm{p}_{\mathrm{T}}^{2} \leq 2(\mathrm{GeV} / \mathrm{c})^{2}$ over a broad range of energy indicates that these effects should be small.

The fact that the $\phi$ meson has the same value of $B$ as observed for the non-strange and strange mesons is significant. This implies that $\phi$ too is dominantly produced through the ozI allowed quark-antiquark (s $\bar{s})$ fusion. This is in accord with the conclusions of Daum et al.(21) and sixel et al.(22).

However, it is well known that the $\mathrm{p}_{\mathrm{T}}$ distributions of the heavy quarkonium states (QD) $J / \psi$ and upsilon are much flatter than those of the light mesons $(23,24)$. This departure from $\mathrm{p}_{\mathrm{T}}^{2}$-universality is due to the fact that whereas the light meson states result from the $\mathrm{q} \vec{q}$ fusion (recombination), the leading contribution to the transverse momentum of the heavy quarkonium states comes from the $2+3$ processes $q \bar{q}+Q \bar{Q} g, g q \rightarrow Q \overline{Q q}$ and $g g \rightarrow Q \bar{Q} g$ (see e.g. ref. 24). These hard processes lead to considerable flattening of the transverse momentum distributions of the $J / \psi$ and upsilon.

Let us assume that the distribution of the primordial $\mathrm{k}_{\mathrm{T}}$ of a quark or an antiquark (assumed to be the same for valence and sea quarks) is gaussian

$$
\mathrm{E}_{\mathrm{q}}\left(\mathrm{k}^{2}=\exp \left(-\mathrm{k}_{\mathrm{T}}^{2} /\left(2 \mathrm{~b}^{2}\right)\right) /\left(2 \mathrm{~b}^{2}\right)\right.
$$

such that

$$
\int_{0}^{\infty} d k_{T}^{2} f_{q}\left(k_{T}\right)=1
$$

This implies that 


$$
\left\langle\mathrm{k}_{\mathrm{T}}^{2}\right\rangle_{\mathrm{q}}=2 \mathrm{~b}^{2}
$$

and $\left\langle k_{T_{q}}=(\pi / 2)^{1 / 2} \mathrm{~b}\right.$

Assuming that the $\mathrm{p}_{\mathrm{T}}^{2}$ distribution of a meson arises as a convolution of two universal distributions of the type (2), one obtains

$$
f_{q \bar{q}}\left(p_{T}\right)=\exp \left(-p_{T}^{2} /\left(4 b^{2}\right)\right) /\left(4 b^{2}\right)
$$

with, $\left\langle k_{T}^{2}\right\rangle_{q \bar{q}}=4 b^{2}$.

Comparing ( 1 ) and (5), and using $B=3.1 \pm 0.2$, we obtain

$$
\begin{aligned}
& b=(4 \mathrm{~B})^{-1 / 2}=0.284 \pm 0.009 \\
& \left\langle k_{T}^{2}\right\rangle_{q \bar{q}}=0.323 \pm 0.021 \\
& \left\langle k_{T_{q}}^{\rangle_{q}}=\sqrt{ } \pi b=0.503 \pm 0.016\right. \\
& \left\langle k_{T}^{2}\right\rangle_{q}=0.162 \pm 0.012 \\
& \left\langle k_{T}\right\rangle_{q}=0.356 \pm 0.011
\end{aligned}
$$

Thus, we conclude that the average intrinsic transverse momentum of the quark is $\left\langle\mathrm{k}_{\mathrm{T}}\right\rangle_{\mathrm{q}}=0.36 \pm 0.01 \mathrm{GeV} / \mathrm{c}$ and the average transverse momentum squared is $\left\langle\mathrm{k}_{\mathrm{T}}^{2}\right\rangle_{\mathrm{q}}=0.16 \pm 0.01(\mathrm{GeV} / \mathrm{c})^{2}$.

\section{Discussion}

We have shown that the universality of the $\mathrm{p}_{T}^{2}$ distribution of the directly produced resonances in soft hadron-hadron processes leads to the conclusion that the average instrinsic transverse 
momentum of the quarks is $\left\langle k_{T}{ }_{q}=0.36 \pm 0.01 \mathrm{GeV} / \mathrm{c}\right.$ and $\left\langle k_{\mathrm{T}}^{2}\right\rangle_{\mathrm{q}}$ $=0.16 \pm 0.01(\mathrm{GeV} / \mathrm{c})^{2}$.

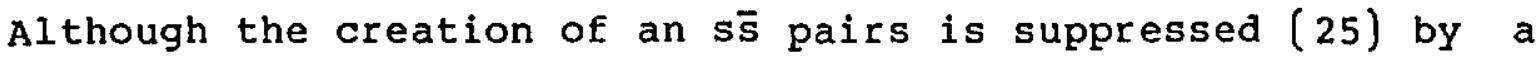
factor $0.29 \pm 0.02$ relative to the uu or da pairs, the intrinsic $<\mathrm{k}_{\mathrm{T}}>$ of the strange quarks appears to have about the same value as that of the non-strange quarks since the transverse momentum distributions of the $K^{*}(890)$ and $\phi$ obey the same universality as observed for the non-strange mesons.

The values of $\left\langle\mathrm{k}_{\mathrm{T}}\right\rangle_{\mathrm{q}}$ and $\left\langle\mathrm{k}_{\mathrm{T}}^{2}\right\rangle_{\mathrm{q}}$ determined above are compared in Table 1 with other measurements reported recently $(26-31)$. These values are lower than those suggested earlier $(1,5)$. Our value is in good agreement with the value reported by Metcalf et al. (26) which is based on the transverse momentum spectrum of low mass muon pairs, $1.4 \leq \mathrm{M}<2.6 \mathrm{GeV}$, produced in the $\pi^{ \pm} \mathrm{N}$ interactions at relatively low energies of 16 and $22 \mathrm{Gev} / \mathrm{c}$ and at low $Q^{2}$.

Berge et al. (27) and Allen et al. (28), (see Table 1), have determined the value of $\left\langle k_{\mathrm{T}}^{2}\right\rangle$ from a measurement of the transverse momenta of the hadrons with respect to the event axis and also with respect to the current directions in $\bar{v} N$ and vp interactions. Tao et al. (29) have obtained $\left\langle\mathrm{k}_{\mathrm{T}}^{2}\right\rangle=0.3(\mathrm{GeV} / \mathrm{c})^{2}$ by studying the transverse momentum distribution of hadrons produced in muon-proton scattering and comparing it to the first order $Q C D$ calculations. It may be remarked that in all these three studies soft gluon contributions have been neglected which in principle could result not only in over-estimation of $\left\langle k_{T}^{2}\right\rangle$ but also make its 
determination somewhat process dependent. The importance of the soft gluons as a source of the transverse momentum is now becoming increasingly apparent as can be seen from the following.

Aubert et al. (30) have studied transverse momentum balance of the final state charged hadrons in $280 \mathrm{GeV} / \mathrm{c} \mu \mathrm{N}$ interactions. In the framework of the first order QCD for hard gluon emission, they require $a\left\langle\mathrm{k}_{\mathrm{T}}^{2} \mathrm{q}_{\mathrm{q}}=0.64(\mathrm{GeV} / \mathrm{c})^{2}\right.$ to $\mathrm{fit}$ the data. However, if they include the soft gluon contributions, the data requires a $\left\langle\mathrm{k}_{\mathrm{T}}^{2}\right\rangle_{\mathrm{q}}$ of only $0.19(\mathrm{GeV} / \mathrm{C})^{2}$, which is consistent with our value.

similar observations can also be made from the analyses of the muon pair production in hadron-hadron interactions. We have recently analysed (7) all the available data on muon pair production in $\mathrm{pN}, \pi^{-} \mathrm{N}$ and $\overline{\mathrm{p}} \mathrm{N}$ interactions, at $\mathrm{p}_{1 \mathrm{ab}}=125$ to $2100 \mathrm{GeV} / \mathrm{c}$, within the framework of order $\alpha_{s}$ QCD assuming a K-factor independent of transverse momentum, and we find that the data require rather large values of $\left\langle k_{\mathrm{T}}^{2}\right\rangle, 0.9$ to $1.5(\mathrm{GeV} / \mathrm{c})^{2}$. on the other hand, Chiappetta and Greco (31), who have analysed pN and $\pi^{-} \mathrm{N}$ dimuon data, obtain good fits to the data with $\left\langle k_{\mathrm{T}}^{2}\right\rangle=0.4(\mathrm{GeV} / \mathrm{c})^{2}$ i.e. $\left\langle\mathrm{k}_{\mathrm{T}}^{2}\right\rangle_{\mathrm{q}}=0.2(\mathrm{GeV} / \mathrm{c})^{2}$, when they include also the soft gluon contribution.

We therefore conclude that all the available observations are consistent with the low value of about $0.36 \mathrm{Gev} / \mathrm{c}$ for the average intrinsic transverse momentum of the quarks as deduced in this study. 


\section{ACKNOWLEDGEMENTS}

We are grateful to Drasko $D$. Jovanovic and the Physics Department of Fermilab for hospitality. We thank Marti Bennett for careful typing.

\section{References}

1. R.P. Feynman, Photon-hadron interactions (Benjamin, New york, 1972);

R.P. Feynman, R.D. Field and G.C.Fox, CALT-68-651 $(1978)$.

2. J. Orear, Cornell University Preprint CLNS 80/446 (1980).

3. L.M. Sehgal, Phys. Lett. 53B (1974) 106.

P.V. Landshoff, Phys. Lett. 66B (1977) 452 .

F.E. Close, F. Halzen and D.M. Scott, Phys. Lett. 68B (1977) 447 .

Satoshi Matsuda, Rutherford Laboratory preprint RL-77-068/A (1977).

J.A. Bartelski, Acta Phys. Polonica B9 (1978) 861 .

4. J. Kogut and L. Susskind, Phys. Lett. 65B (1976); Phys. Rev. D9 (1974) 697; ibid D9 (1977) 3391.

5. M. Della Negra et al., Nucl. Phys. B127 (1977) 1.
A.P. Contogouris,
R. Gaskell
and S. Papadopoulos,

Phys. Rev. D 17 (1977) 2314.

R.D. Field, Phys. Rev. Lett. 40 (1977) 997.

6. K. Kajantie, J. Lindfors and R. Raitio, Phys. Lett. $74 B$ (1978) 384 .

G. Altarelli, G. Parisi and R. Petronzio, Phys. Lett. 76B (1978) 351 .

7. P.K. Malhotra, Proc, of Workshop on Drell-Yan Processes, Fermilab, October 7-8, 1982 .

B. Cox and P.K. Malnotra, to be published. 
8. W.E. Caswell, R.R. Horgan and S.J. Brodsky, SLAC preprint SLAC-PUB-2106 (1978).

R.R. Horgan and P.N. Scharbach, Phys. Lett. 81B (1979) 215 .

9. P.K. Malnotra, Proc. VII Int. Colloguium on Multiparticle Reactions, Tutzig (1976) 253.

M. Deutschmann et al., Nucl. Phys. B103(1976) 426 .

J. Bartke et al., Nucl. Phys. B107 (1976) 93.

H. Kirk et al., Nucl. Phys. B128 (1977) 397.

10. W. Kittel, VIII Int. Symposium on Multiparticle Dynamics, Kayserberg (1977) A-81.

11. P.K. Malhotra, to be published.

12. H. Kirk et al., Nucl. Phys. B116 (1976) 99.

13. H. Grassler et al., Nucl. Phys. B132 (1978) 1.

14. R. Hamatsu et al., Bombay-CERN-College de France-Madrid Collaboration, Nucl. Phys. B123 (1978) 189.

15. A.M. Cooper et al., Bombay-CERN-College de France-Madrid Collaboration, Nucl. Phys. B136 (1978) 365 .

16. D. Drijard et al., Z. Phys. C9 (1981) 293.

17. B.M. Whyman et al., Z. Phys. C12 (1982) 203.

18. T. Akesson et al., CERN-EP/82-01 (1982).

19. R. Gottgens et al., Z. Phys. C12 (1982) 323 .

20. V.V. Anisovich and V.M. Shekhter, Nucl. Phys. B55 (1973) 455 .

V.M. Shekhter and L.M. Shcheglova, Sov. J. Nucl. Phys. 27 (1978) 567 .

21. C. Daum et al., Nucl. Phys. B186 (1981) 205.

22. P. Sixel et al. CERN/EP 82-7 (1982).

23. Yu.M. Antipov et al., Phys. Lett 76B (1978) 235 .

K.J. Andersson et al., Phys. Rev. Lett. 42 (1979) 944.

C. Kourkoumelis et al., Phys. Lett. 91B (1980) 29.

P. Sixel et al. CERN/EP 82-7 (1982). 
24. 2. Kunszt, E. Pietarinen and E. Reya, Phys. Rev. D21 (1980) 733 .

25. P.K. Malhotra and R. Orava, "Measurement of strange quark suppression in hadronic vacuum", FERMILAB-Pub-82/79 $(1982)$.

26. W. Metcalf et al., Phys. Lett. 91B (1980) 275.

27. J.P. Berge et al., Nucl. Phys. B203 (1982) 16.

28. P. Allen et al., Nucl. Phys. B188 (1981) 1.

29. C. Tao et al., Phys. Rev. Lett. 44 (1980) 1726 .

30. J.J. Aubert et al., EMC, "Balance of transverse momentum in $\mu N$ scattering", contributed paper, XXI Int. Conf. on High Energy Physics, Paris, 1982.

31. P. Chiappetta and M. Greco, Phys, Lett. 106B (1981) 219.

Table 1: A compilation of recent determinations of the intrinsic transverse momentum of quarks.

\begin{tabular}{|c|c|c|c|c|}
\hline Process & $\begin{array}{l}\sqrt{\mathrm{s}}, \mathrm{W} \\
(\mathrm{GeV})\end{array}$ & $\begin{array}{l}\left\langle\mathrm{k}_{\mathrm{T}}^{2}\right\rangle_{\mathrm{q}} \\
(\mathrm{GeV} / \mathrm{c})^{2}\end{array}$ & $\begin{array}{l}\left\langle\mathrm{k}_{\mathrm{T}}\right\rangle_{\mathrm{q}} \\
(\mathrm{GeV} / \mathrm{c})\end{array}$ & Reference \\
\hline$h h \rightarrow M X$ & & $0.16 \pm 0.01$ & $0.36 \pm 0.01$ & This work \\
\hline$\pi^{-} N+\mu^{+} \mu^{-} X$ & $1.4-2.6$ & - & $0.32 \pm 0.04$ & 26 \\
\hline$\nabla N+\mu^{+} n X$ & 10 & $0.4 \pm 0.1$ & $0.56 \pm 0.07$ & 27 \\
\hline$\nu p+\mu-h X$ & $>4$ & $0.30 \pm 0.05$ & $0.49 \pm 0.04$ & 28 \\
\hline$\mu p+\mu h x$ & $>10$ & 0.30 & 0.49 & 29 \\
\hline$\mu N \rightarrow \mu h X$ & $10-18$ & 0.19 & 0.39 & 30 \\
\hline$h N \rightarrow \mu^{+} \mu^{-} X$ & -- & 0.20 & 0.40 & 31 \\
\hline
\end{tabular}

